\title{
Calcified Liver Metastases from a Neuroendocrine Tumor of the Lung (Atypical Carcinoid) - A Case Report
}

\section{(6)(1) (\$)}

\section{Introduction}

Pulmonary neuroendocrine tumors (NETs) are rare tumors with an incidence rate of $0.2-2 / 100000$ population/year in Western countries (M. E. Caplin et al. Ann Oncol 2015; 26:1604-20). They account for $1-2 \%$ of all neoplasms of the lung and constitute one-fourth to one-third of all NETs. Atypical carcinoids are far less common than typical carcinoids and predominantly occur in male smokers aged $50-70$ years. Most pulmonary NETs are asymptomatic due to their peripheral location. Surgical resection is the treatment of choice. Medical management should take hormone-related symptoms into account.

To the best of our knowledge, this is the first report of a pulmonary NET with unusual calcified liver metastases. Diagnostic workup of the patient included ultrasound, computed tomography $(\mathrm{CT})$, magnetic resonance imaging (MRI), and ultrasound-guided biopsy of the liver lesions.

\section{Case Description}

A 75-year-old woman with visual impairment of the right eye was admitted to the Department of Ophthalmology of our hospital. She had a history of type II diabetes mellitus, partial strumectomy and arterial hypertension. The clinical examination and complete blood count were normal. Her medications included levothyroxine, triamterene, hydrochlorothiazide, and pantoprazole. Optical coherence tomography revealed a choroidal lesion in the right eye. The appearance was highly suspicious for choroidal melanoma.

A chest radiograph and abdominal ultrasound were performed as part of the routine staging of choroidal melanoma. The chest radiograph demonstrated a nodule in the middle lobe of the right lung. The abdominal ultrasound examination revealed multiple hyperechoic non-vascularized lesions in both hepatic lobes (Siemens Acu- son S2000, 4C1 transducer). In segment III, the lesions were up to $4 \mathrm{~cm}$ in size and had hypoechoic margins, partially with posterior acoustic shadowing ( $\triangleright$ Fig. 1). For further workup of the pulmonary nodule and the calcified liver lesions, a CT of the chest and abdomen and MRI of the upper abdomen were performed ( $>$ Fig. 2; CT: Toshiba Aquilion Prime 80; MRI: Siemens Magnetom Aera 1.5 Tesla). CT demonstrated a single mass with smooth margins $(16 \times 20 \mathrm{~mm})$ in the lung, suggesting malignancy, and additionally revealed liquefied and partially calcified lymph node metastases below the carina and in the right hilum. T2-weighted MR images revealed high-signal-intensity lesions in both liver lobes with early arterial ring enhancement ( $>$ Fig. 2 ), characterizing the calcified liver lesions as metastases. In summary, imaging workup showed malignant lesions of the choroid, lung, and liver as well as metastatic hilar and mediastinal lymph nodes ( $>$ Fig. 3).

For further clarification, an ultrasoundguided percutaneous biopsy of the liver metastases was performed (Bard TruCut biopsy needle, $18 \mathrm{G}, 160 \mathrm{~mm}$ ) after obtaining written informed consent.

\section{Pathology}

No melanin production was demonstrated in the biopsy tissue. Histology revealed liver metastases from an NET (G2 according to WHO), most likely from a primary in the lung (atypical carcinoid). The differential di-

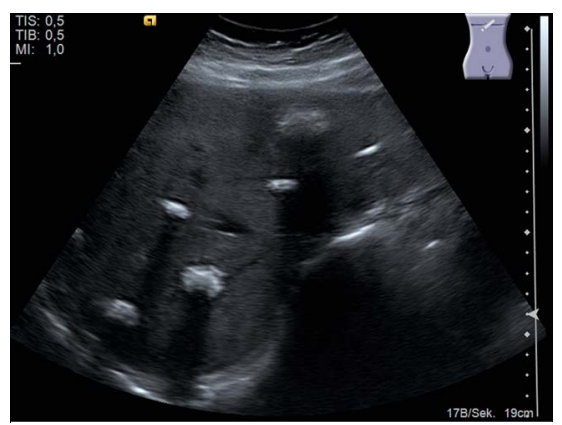

- Fig. 1 Ultrasound of the liver showing hyperechoic calcified liver metastases with posterior acoustic shadowing. agnosis included choroidal and gastrointestinal NET. However, the pattern of metastatic spread made these primary tumor sites unlikely.

\section{Discussion}

To the best of our knowledge, this is the first report of a pulmonary NET with unusual calcified liver metastases. A PubMed database search (search terms: calcified metastases, pulmonary NET, calcified liver metastases, hepatic calcifications) revealed no case of pulmonary NET with calcified liver metastases. Our search revealed only a single case of a patient with pancreatic NET and calcified liver metastases (T. Kawamoto et al. Clin J Gastroenterol 2014;7:460-4).

The sonographic appearance of the liver lesions did not allow definitive characterization as benign or malignant. Their appearance was consistent with hepatic metastases, calcified adenomas, and hepatic tuberculosis. Further workup with CT and MRI allowed definitive classification of the lesions as calcified metastases. The ultrasound-guided liver biopsy was crucial for identifying the primary tumor. Otherwise multiple calcified liver metastases would primarily suggest metastatic spread from a mucin-producing primary tumor, for example in the colon, breast, stomach, or ovaries (C. Stoupis et al. Radiographics 1998;18:675-85).

We did not perform contrast-enhanced ultrasound (CEUS) in our patient because of the multiplicity of liver lesions and acoustic shadowing due to calcification of the lesions. It is known from previous studies that the detection and characterization of hepatic lesions, e.g., metastases from colorectal cancer, can be significantly improved by CEUS compared to non-enhanced ultrasound (V. Cantisani et al. Ultraschall Med 2010;31:500-5, M. D’Onofrio et al. Radiol Med 2008;113:76-86).

In the case presented here, imaging findings and liver biopsy ruled out the initially suspected choroidal melanoma, leading instead to the diagnosis of a pulmonary NET with metastatic spread to the liver, 

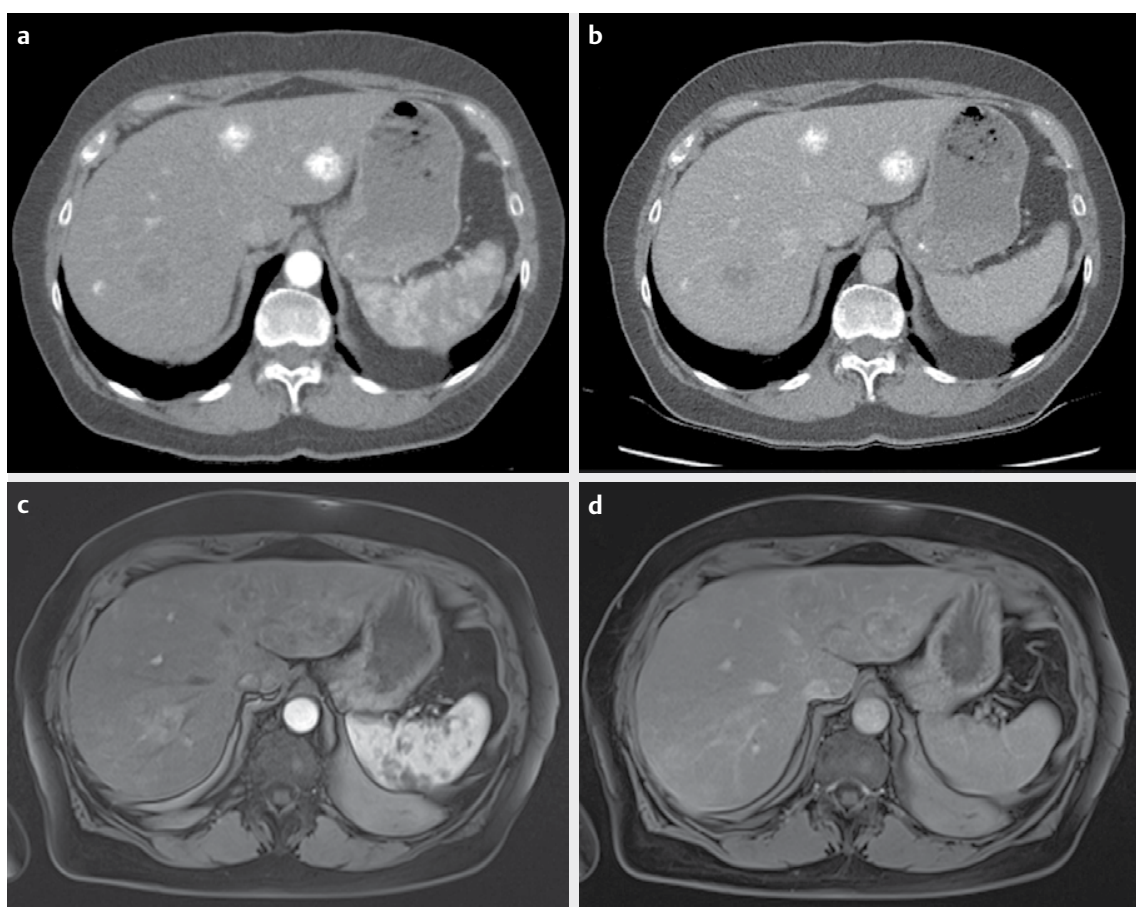

- Fig. 2 Upper row: Contrast-enhanced CT showing calcified liver metastases: a arterial phase and $\mathbf{b}$ venous phase. Lower row: T1-weighted contrast-enhanced MR images: $\mathbf{c}$ hyperintense enhancement in arterial phase $\mathbf{d}$ becoming less distinct in portal venous phase. The calcifications are not apparent on the MR images.

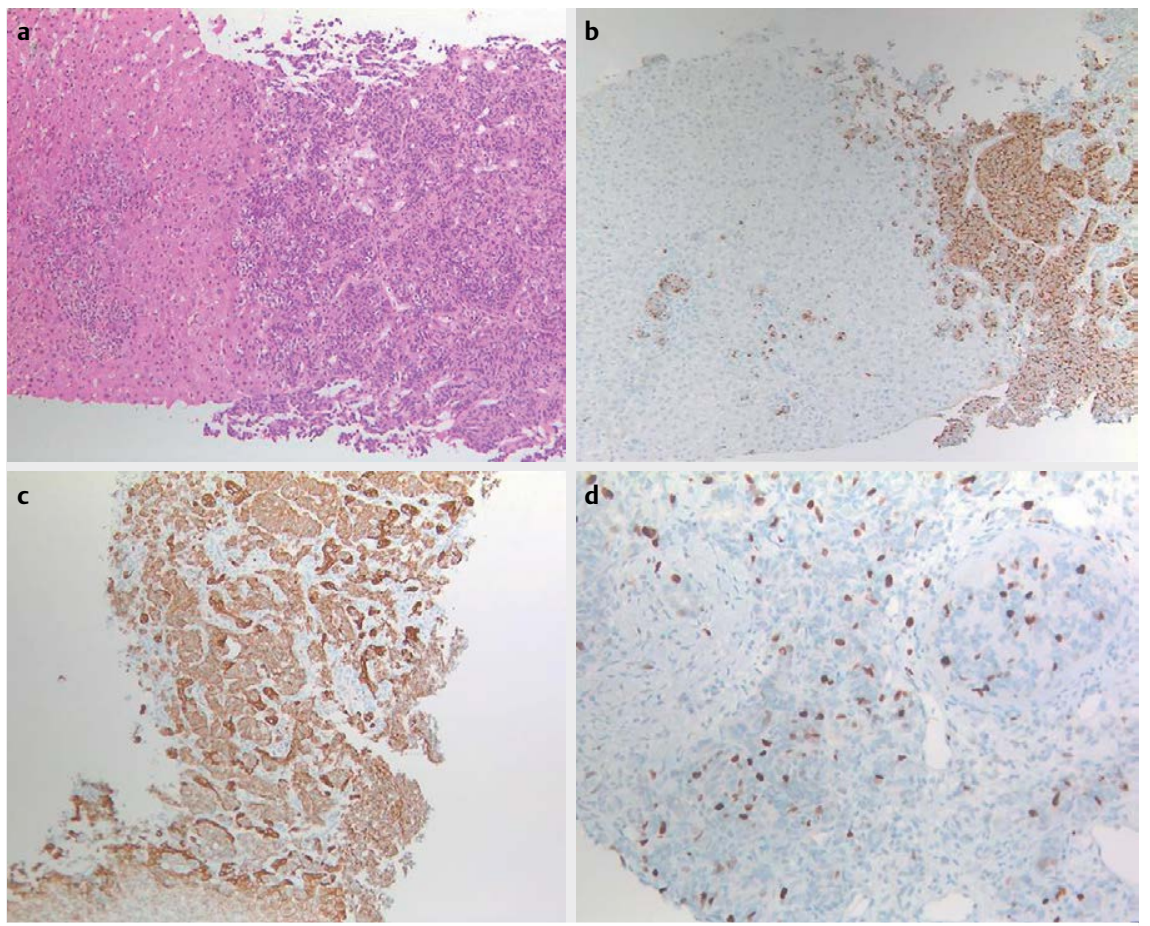

- Fig. 3 a Histologic examination of the liver biopsy revealed infiltration by a solid tumor with a nest-like growing pattern composed of isomorphic tumor cells with ill-defined cell borders. $\mathbf{b}$ Immunohistology of the tumor cell infiltrates demonstrated expression of synaptophysin as well as chromogranin and pancytokeratin. $\mathbf{c}$ Islet 1 revealed only nonspecific staining of hepatocyte cytoplasm. $\mathbf{d}$ The Ki-67 proliferation rate was variable. The integral count of Ki-67 positive cells was $15 \%$. choroid and lymph nodes. Workup was initiated based on this diagnosis.

However, the cause of calcification of liver and lymph node metastases in our patient remains unclear. At the time of diagnosis, no hypercalcemia was apparent $\left(\mathrm{Ca}^{2+}=2.4 \mathrm{mmol} / \mathrm{l}, \mathrm{n}=2.2-2.5 \mathrm{mmol} / \mathrm{l}\right)$. Therefore, metabolic causes or a paraneoplastic syndrome with calcification seems unlikely. We hypothesize that rapid growth of the metastases led to necrosis with subsequent dystrophic calcification.

\section{Conflict of Interest}

The authors declare that they have no conflict of interest.

\section{Authors}

Rolf Reiter ${ }^{1}$, Jochen Maul², Jan Christian Preis ${ }^{3}$, Hendrik Blaeker ${ }^{4}$, Zarko Grozdanovic ${ }^{1}$

1 Charité - Universitätsmedizin Berlin, Department of Radiology, Berlin, Germany

2 Charité - Universitätsmedizin Berlin, Interdisciplinary Ultrasound Unit, Berlin, Germany

3 Charité - Universitätsmedizin Berlin, Department of Gastroenterology, Berlin, Germany

4 Charité - Universitätsmedizin Berlin, Department of Pathology, Berlin, Germany

\section{Correspondence}

\author{
Dr. Rolf Reiter, MD \\ Charité - Universitätsmedizin Berlin \\ Department of Radiology \\ Hindenburgdamm 30 \\ Charité Campus Benjamin Franklin \\ 12203 Berlin \\ Germany \\ Tel.: +49/30/84453041 \\ rolf.reiter@charite.de
}

\section{Bibliografie}

DOI http://dx.doi.org/10.1055/s-0043-100504 Ultrasound Int Open 2017; 3: E89-E90

(C) Georg Thieme Verlag KG Stuttgart · New York ISSN 0722-1819 